\title{
Osteoporosis and inflammation
}

\author{
Osteoporose e inflamação
}

Paulo Gustavo Sampaio Lacativa', Maria Lucia Fleiuss de Farias ${ }^{2}$

\section{SUMMARY}

Several inflammatory diseases such as rheumatoid arthritis, systemic lupus erythematosus, inflammatory bowel disease, celiac disease, cystic fibrosis and chronic obstructive pulmonary disease have been associated to bone resorption. The link between osteoclast, macrophage colony stimulating factor and pro-inflammatory cytokines, especially tumor necrosis factor- $\alpha$ and interleukin-1 explain the association between inflammation and osteoporosis. These diseases are related to osteoporosis and high fracture risk independent of other risk factors common to inflammatory diseases such as reduced physical activity, poor nutritional status, hypovitaminosis $D$, decrease in calcium intake and glucocorticoid treatment. Erythrocyte sedimentation rate and $\mathrm{C}$-reactive protein should always be performed, but the indication about when to perform the densitometry test should be analyzed for each disease. Bisphosphonates are nowadays the best choice of therapy but new medications such as denosumab, IL-1 receptor antagonist, and TNF- $\alpha$ antibody have risen as new potential treatments for osteoporosis secondary to inflammation. Arq Bras Endocrinol Metab. 2010;54(2):123-32

\section{Keywords}

Osteoporosis; inflammation; arthritis, rheumatoid; lupus erythematosus, systemic; interleukin-1; tumor necrosis factor-alpha

\section{SUMÁRIO}

Diversas doenças inflamatórias têm sido associadas à reabsorção óssea, como a artrite reumatoide, o lúpus eritematoso sistêmico, a doença inflamatória intestinal, a doença celíaca, a fibrose cística e a doença pulmonar obstrutiva crônica. A ligação entre osteoclastos, fator estimulador de colônia de macrófagos e citocinas pró-inflamatórias, principalmente o fator de necrose tumoral- $\alpha$ e interleucina-1, explica a associação entre a inflamação e a osteoporose. Essas doenças estão relacionadas com osteoporose e aumento do risco de fratura, independentemente de outros fatores de risco comuns às doenças inflamatórias, tais como redução da atividade física, estado nutricional, hipovitaminose $D$, diminuição da ingestão de cálcio e uso de glicocorticoides. A velocidade de hemossedimentação e proteína C-reativa devem ser sempre realizadas, mas a indicação do exame de densitometria óssea deve ser analisada em cada doença. Os bisfosfonatos são atualmente a melhor opção de terapia, mas novos medicamentos, tais como denosumabe, antagonista do receptor de IL-1 e anticorpos anti-TNF- $\alpha$, surgem como novos potenciais tratamentos para a osteoporose secundária à inflamação. Arq Bras Endocrinol Metab. 2010;54(2):123-32

\section{Descritores}

Osteoporose; inflamação; artrite reumatoide; lúpus eritematoso sistêmico; interleucina-1; fator de necrose tumoral-alfa

\section{INTRODUCTION}

Qeveral inflammatory diseases have been associated $\checkmark$ to bone resorption. Rheumatoid arthritis (RA), for example, is associated with focal bone erosion at the margins of the affected joint. Periodontal disease, a chronic inflammation of the gums, is associated with destruction of the alveolar bone of the jaw. Moreover, osteoporosis
${ }^{1}$ Departamento de Medicina Interna, Hospital Universitário Pedro Ernesto, Universidade do Estado do Rio de Janeiro (Uerj), Rio de Janeiro, RJ, Brasil 2 Serviço de Endocrinologia, Departamento de Medicina Interna, Hospital Universitário Clementino Fraga Filho, Universidade Federal do Rio de Janeiro (UFRJ), Rio de Janeiro, RJ, Brasil

Correspondence to:

Paulo Gustavo Sampaio Lacativa Rua Casuarina, 135/401 22261-160 - Rio de Janeiro, RJ, Brasil pglaca@yahoo.com

Received on Dec/12/2009 Accepted on Feb/28/2010 and fragility fractures are more prevalent in RA, systemic lupus erythematosus (SLE), inflammatory bowel disease (IBD), chronic obstructive pulmonary disease (COPD) and other inflammatory diseases, when compared to the healthy population. These findings lead to the link between osteoclasts, the unique cells responsible for bone loss, and pro-inflammatory cytokines (1). 
Other risk factors for osteoporosis are present in inflammatory disease besides inflammation per se, and should be assessed and corrected, such as reduced physical activity due to fatigue, muscle waist or painful joint lesions that can be so severe that they result in immobilization; poor nutritional status secondary to malabsorption or increased basal energy expenditure, that can lead to hypovitaminosis $\mathrm{D}$ and decrease in calcium intake; and glucocorticoid (GC) treatment, among others.

Finally, bisphosphonates are well established for treatment of osteoporosis secondary to inflammatory disease and/or GC, but the understanding of the interplay of inflammation and osteoclast can lead to the development of new drugs for prevention and treatment of bone loss found in those patients.

\section{PATHOPHYSIOLOGY}

All diseases involving bone loss have a common pattern: the osteoclast is the cell exclusively responsible for bone resorption (2), and osteoporosis occurs when osteoclast activity overcomes osteoblast activity (3).
Inflammation modulates bone resorption mainly by two mechanisms. Firstly, pro-inflammatory cytokines have a final common mediator of osteoclast function: receptor activator of nuclear factor-B (RANK) and its functional ligand (RANKL), also known as TRANCE (TNF-related activation induced cytokine) (Figure 1). Secondly, osteoclastogenesis can be regulated through the modulation of macrophage colony stimulating factor (M-CSF) (Figure 2).

\section{RANK/RANKL}

RANKL is a membrane-bound tumor necrosis factor (TNF) receptor expressed on osteoblast precursor cells that recognize RANK on the osteoclast surface through a direct cell-cell interaction. This process is essential for osteoclast differentiation, activation and survival. RANKL is considered the key osteoclastogenic cytokine as the RANKL-RANK interaction stimulates several transcription factors and all three families of MAP kinases. Moreover, RANKL induces expression of the c-Fos family and promotes nuclear translocation of Jun proteins and NFATcl. Most importantly, deletion or inactivation of c-Fos, c-Jun, or NFATcl results in failed osteoclast differentiation and severe osteopetrosis (3).

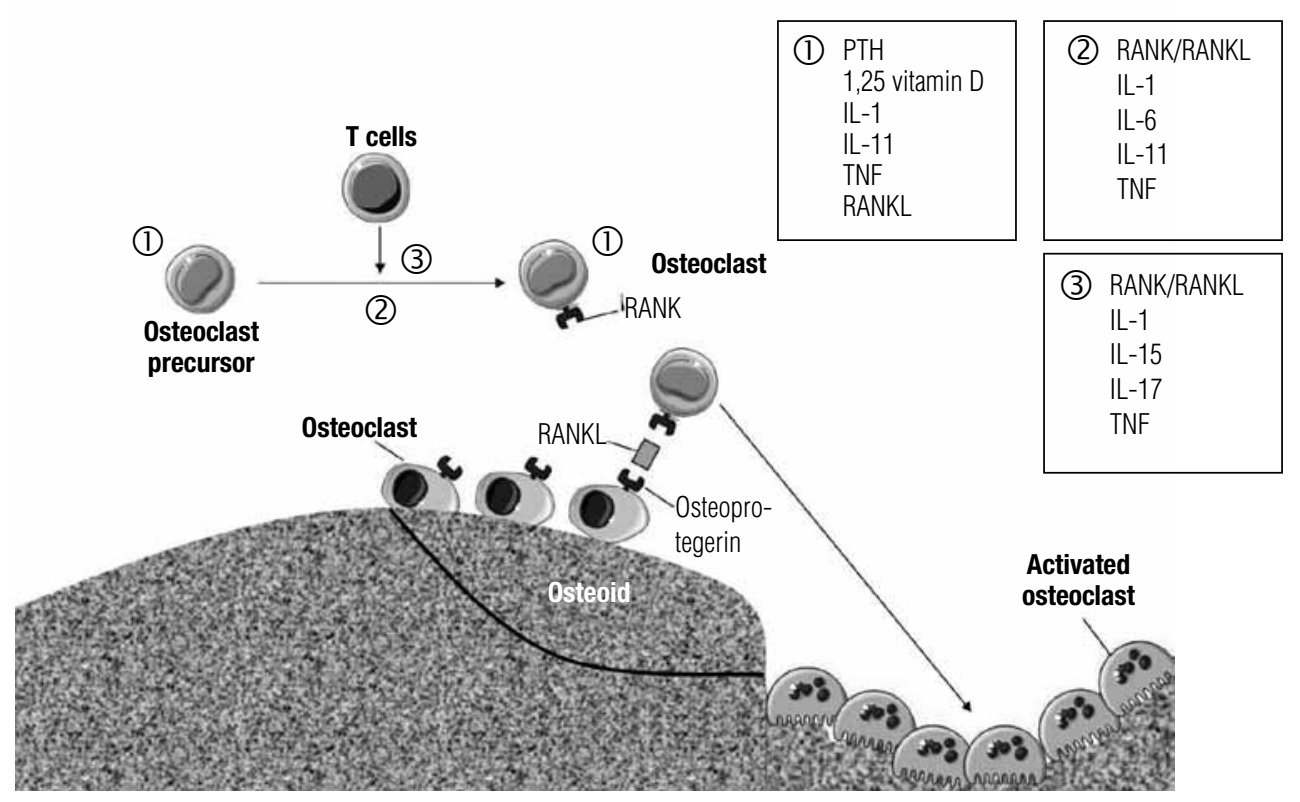

Figure 1. RANK/RANKL/OPG system and its relation with proinflammatory cytokines. Maturation of osteoclast precursor and activation of mature osteoclast are stimulated by several factors (Text Box 1). Activated T-lymphocyte cells (T-cells) produce substances that stimulate osteoclast maturation such as receptor activator of NFKB ligand (RANKL), tumor necrosis factor (TNF) and other interleukins (ILS) (Text Box 3). Mature osteoclasts express RANK on its surface, and when RANK binds to RANKL, osteoclasts become activated. Osteoprotegerin acts as a competitor for RANK binding, affecting the interaction RANK-RANKL. Activated osteoclast acts causing bone resorption. Adapted from reference 4. 


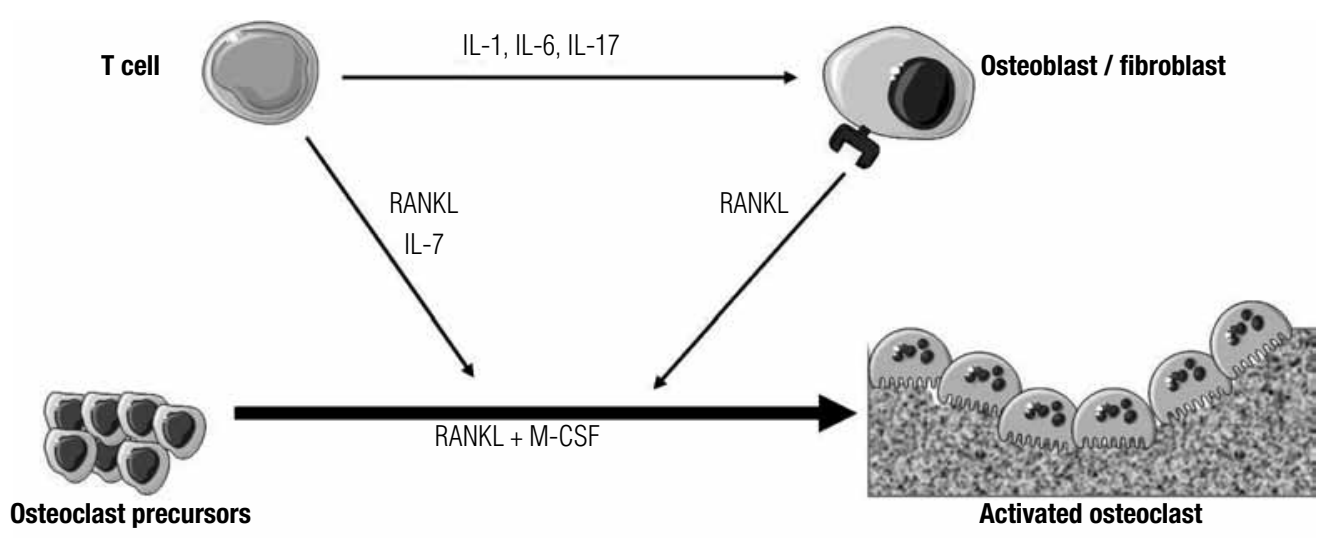

Figure 2. Mechanisms by which inflammation modules osteoclast and bone resorption. Cells of the myelomonocytic lineage (osteoclast progenitor) under the influence of macrophage-colony stimulating factor (M-CSF) and receptor activator of NFkB ligand (RANKL) differentiate into osteoclasts. T-lymphocytes (T-cell) promote osteoclast formation directly by production of IL-7 and RANKL. T-cells also produce several pro-inflammatory cytokines (IL-1, IL-6, IL-17) that promote osteoblast and fibroblast production of RANKL, that also stimulates osteoclast formation. Adapted from reference 5.

Osteoprotegerin (OPG) ligand is a membrane-bound TNF-related factor that acts as an endogenous inhibitor for RANKL specifically binding it and blocking its interaction with RANK (Figure 1). OPG, like RANKL, is produced by osteoblast lineage cells (1). Mice lacking functional RANKL develop a form of osteopetrosis and lack osteoclasts, despite the presence of osteoclast precursors (1). Conversely, OPG knockout mice develop osteopenia with an increased number of activated osteoclasts present. (1). Therefore the OPG/ RANKL ratio dictates the rate of bone resorption in a number of pathological states, including inflammatory diseases (3).

\section{Macrophage colony stimulating factor}

A clear relationship between inflammation and osteoporosis is the demonstration that the principal osteoclast precursor is located in the bone marrow as it is a member of the monocyte/macrophage family (4). $\mathrm{M}$-CSF produced by marrow stromal cells is essential for macrophage survival and proliferation. M-CSF binds to its receptor c-Fms on precursor cells enhancing the number of precursor cells that will differentiate into osteoclast via RANKL action (Figure 2). In fact, pure populations of osteoclasts in vitro are achieved by culturing marrow macrophages in the presence of only RANKL and M-CSF $(3,5)$. As an example of the importance of these mechanisms, mice which lack functional M-CSF present with osteopetrosis (3).

\section{Pro-inflammatory cytokines}

One of the first studies to support the relation between cytokines and osteoclast activity demonstrated in vitro that exposure to activated leukocyte supernatant was associated with increased osteoclast number and function. The authors concluded that probably there are one or more factors produced by activated leukocytes that induce osteoclast formation and activation (1). These factors are now known as pro-inflammatory cytokines produced by macrophages and lymphocytes, the largest and most predominant source of cell-derived regulatory signals in the body. Since then, several cytokines have been related to osteoclast bone resorption: TNF- $\alpha$, interleukin (IL)-1, IL-6, IL-11, IL-15 and IL-17 (1).

\section{Tumor necrosis factor $\alpha$}

TNF- $\alpha$ is expressed by T-lymphocytes ( 5 ) and promotes bone loss in conditions such as inflammatory osteolysis, alveolar bone loss in periodontal inflammation, and postmenopausal osteoporosis. It is probably the key cytokine mediating the periarticular bone loss of RA (3).

There are two mechanisms by which TNF- $\alpha$ acts in osteoclasts, both marrow stromal cells and osteoclast precursors express TNF- $\alpha$ receptors. The main process occurs when stromal cells are exposed to TNF- $\alpha$ and produce RANKL, M-CSF, and IL-1, which promote osteoclast formation and activation. TNF- $\alpha$ and RANKL are synergistic, and minimal levels of one markedly enhances the osteoclastogenic capacity of the other (3). TNF- $\alpha$ also has potent antiapoptotic effects on osteoclasts, prolonging their lifespan (6). The second mechanism occurs when the inflammatory process becomes more aggressive and TNF- $\alpha$ may promote osteoclast formation by directly stimulating its precur- 
sors in the absence of stromal cells responsive to the cytokine, perhaps through activation of transforming growth factor (TGF)- $\beta$ (3). Mice whose CD4 cells do not respond to TGF- $\beta$ have a lower bone mineral density (BMD) than wild-type animals (5).

Another important role of TNF- $\alpha$ in inflamed tissue is the capacity to induce intercellular adhesion molecule (ICAM-1) in endothelial cells. This molecule binds with circulating leukocytes in vessels, resulting in accumulation of lymphocytes which will produce more TNF- $\alpha$ and resulting in a self-feeding circle. Activated T-cells besides expressing RANKL also adhere to osteoblasts and induce adhesion-dependent osteoclast maturation (7).

\section{Interleukin-1}

The major source of IL-1 is macrophages and this cytokine plays an important role in osteoclast action through some pathways. Firstly, when acting on stromal cells TNF- $\alpha$ stimulates IL-1 production which mediates a substantial component of TNF- $\alpha$ 's osteoclastogenic effect. The intimate relationship between these two cytokines is reflected by the fact that optimal arrest of inflammatory osteoclastogenesis and bone destruction requires blockade of both (3).

IL-1 also stimulates osteoclast activity by increasing production of M-CSF (1), but it enhances osteoclastogenesis only in the presence of permissive concentrations of RANKL (3). Again, as with TNF- $\alpha$, IL- 1 also inhibits osteoclast apoptosis and induces ICAM (6).

\section{Other pro-resorptive interleukins}

Activated T-lymphocytes produce IL-1 and other pro-resorptive cytokines including IL-6, IL-11, IL-15 and IL17, each of which can stimulate RANKL expression (5).

T-lymphocytes that produce IL-17 are designated as Th17 cells which also express TNF- $\alpha$, IL-6 and MCSF. Proliferation and survival of Thl7 cells is regulated by IL-23. The importance of IL-17 and IL-23 in RA and other inflammatory diseases has been highlighted in studies using knockout mice. IL-17 or IL-23 deficient mice were resistant to experimental collageninduced arthritis. Therefore, Thl7 cell function is an important immunomodulator of osteoclastic bone resorption (5).

IL- 6 and IL- 11 are influenced by bone-active systemic hormones (such as estrogen, parathyroid hormone $(\mathrm{PTH})$, 1,25-dihydroxyvitamin $\mathrm{D}_{3}$ and thyroxine), which exert their potent influences on bone remodeling and skeletal homeostasis by regulating the production and action of these cytokines (8).

T-lymphocytes also produce IL-7 but this cytokine supports osteoclast formation directly, mediated by a RANKL-independent process (6).

\section{Glucocorticoid use}

In all inflammatory diseases, use of glucocorticoids (GC) is a common therapy. There is no doubt about the deleterious effect of GC in bone metabolism, suppressing bone formation and enhancing bone resorption. The addition of GC to osteoprogenitor cells in vitro actually increases their bone-forming capacity but it also increases apoptosis of mature osteoblasts and osteocytes and therefore affects capacity of bone formation. This potent anti-inflammatory drug reduces production of pro-inflammatory cytokines (IL-1, IL-6, and TNF- $\alpha$ ), but provokes bone resorption by increasing the synthesis of RANKL and inhibiting OPG production with consequent induction of osteoclastogenesis. This increased bone resorption can explain the response to antiresorptive drugs in the management of GC-induced osteoporosis. GC also inhibit calcium absorption in the gastrointestinal tract and induce renal calcium loss. Other significant effects in bone metabolism is due to vitamin D catabolism and consequent deficiency induced by this drug (9).

There is plenty of evidence about the deleterious effects of GC, with a strong correlation between cumulative dose and bone loss as well as between daily dose and fracture risk. The risk of fracture increases rapidly after 3 to 6 months of the onset of GC use and decreases after stopping therapy (9).

In conclusion, in inflammatory diseases the effect of systemic inflammation as well as the deleterious effects of GC use must be valorized in order to adequate prevention and treatment of osteoporosis.

\section{RHEUMATOID ARTHRITIS}

Rheumatoid Arthritis (RA) is the most common chronic autoimmune disease there is. It is characterized by inflammation of the synovium that occurs with activation and proliferation of synoviocytes, underneath which macrophages, lymphocytes, plasma cells and dendritic cells infiltrate and new blood vessels proliferate. "Pannus" a result of this inflammatory process invades the 
articular cartilage, causing bone erosions, periarticular osteoporosis, and finally, joint destruction (10).

Lymphocyte and synovial cell expansion occur prior to bone destruction suggesting that these cells are responsible for osteoclast formation and activation and therefore for juxta-articular bone loss. Activated Tlymphocytes express RANKL $(2,3,5)$ and produce the pro-inflammatory stimuli IL-1, IL-17, TNF- $\alpha$ and PGs that together with ICAM-1 create the disequilibrium in bone metabolism by favoring bone resorption $(7,10)$. There is a direct correlation between serum OPG, erythrocyte sedimentation rate and a score of disease activity in patients with RA. The rise in OPG probably represents a response to reverse RANKL increased (11). IL-6 concentration in the bone marrow is also related to systemic and periarticular bone loss in these patients (8).

Systemic inflammation and GC use accelerates bone loss independent of other risk factors (12) but other factors must be valorized. Immobilization due to pain from inflamed joints, impairment of physical activity, reduced calcium intake, and poor nutrition associated with enhanced basal energy expenditure are also risk factors related to low BMD, common is this population. In children, pubertal delay and growth stunting is common which can negatively affect bone modeling (10).

Consequently, increase in bone resorption, both focal and systemic, are common in patients with RA (10). In patients with active RA compared to matched controls and patients with inactive RA serum osteocalcin which reflects bone formation was found to be significantly lower and crosslinked $\mathrm{N}$-telopeptidases of type $\mathrm{I}$ collagen (NTX) and deoxypyridinoline (DPD) which reflect bone resorption, were significantly higher. There were positive correlations between these bone markers and disease activity (13). The prevalence of low bone mass in children with juvenile idiopathic arthritis (JIA) varies from $25 \%$ to $40 \%$. Finally, fracture risk at both vertebral and non-vertebral sites is increased (10).

\section{SYSTEMIC LUPUS ERYTHEMATOSUS}

Systemic lupus erythematosus (SLE) is a systemic autoimmune inflammatory disorder. Serum TNF- $\alpha$, IL- 1 and IL-6, are increased in patients with SLE. The high homocysteine concentration and high levels of LDL oxidation products present in these subjects due to the inflammation process can also contribute to osteopotorosis. $(10,14)$.
Prevalence of osteoporosis in SLE patients is between $4 \%$ and $22 \%$ (15). Bone loss is most prominent in the lumbar spine and is much less evident at the hip joint and can be present at diagnosis prior to the initiation of treatment involvement (14). In children, the prevalence of low lumbar spine BMD is approximately $40 \%$ (10). Patients with SLE have a significantly higher risk of vertebral fractures than controls and the majority of these fractures occur in premenopausal women (14). Borba and cols. (15) found $21.4 \%$ of vertebral fractures in SLE premenopausal patients, and none in the matched control group of similar age. The presence of the fractures was not related to BMD or GC use.

Risk factors that contribute to osteoporosis in patients with SLE are, besides the inflammatory process and GC use, reduced physical activity or immobility, hypovitaminosis D secondary to avoidance of sun exposure (due to photosensitivity) and/or due to chronic renal failure, premature menopause or hypogonadism induced by cyclophosphamide and gonadotropin-releasing-hormone agonists $(10,14,16)$. Borba and cols. (17) studied 36 patients with SLE compared to placebo and concluded that SLE patients demonstrated changes in bone remodelling and a high prevalence of vitamin D deficiency. Both of them were related to disease activity indicating the need for adequate vitamin D replacement.

\section{INFLAMMATORY BOWEL DISEASE}

The prevalence of osteopenia and osteoporosis varies between $22 \%-77 \%$ and $14 \%-41 \%$ of patients with IBD (18), although inflammatory bowel disease (BMD) changes are not excessive and not consistent in literature (11). The risk of hip fracture is increased by $86 \%$ in patients with Crohn's disease and by $40 \%$ in patients with ulcerative colitis (11). Children with inflammatory bowel disease (IBD) gain bone mass, but the rate of bone mineral accrual is slower than normal, until at least 2 years after diagnosis. Catch-up growth may not occur in children with IBD and inadequate linear growth may stunt the normal acquisition of bone mineral (10).

Inflammatory process and GC therapy are not the only causes of low BMD, but also malabsorption and malnutrition, restriction diets (that include low vitamin $\mathrm{D}$ and calcium intake), hypovitaminosis $\mathrm{D}$ and immobilization $(19,20)$. Souza and cols. (21) measured 25 hidroxivitamin D of 76 patients with IBD (37 with ulcerative colitis and 39 with Crohn's disease) and revealed that all of them have lower concentrations compared 
to the control group, and almost half the patients have concentrations that classified them as vitamin D deficient. The concentration of 25 hidroxivitamin $\mathrm{D}$ was related to BMD.

The IL-2 deficient mouse model of colitis is known to develop both osteopenia and colitis, but low bone mass does not occur in T-cell deficient rats, indicating the central role of activated T-cells in bone loss in IBD models (22). High levels of inflammatory cytokines (TNF- $\alpha$, IL-1, IL-6, IL-11, IL-17, TGF- $\alpha$, epidermal growth factor, and prostaglandin E2) are detected in the gut mucosa and in the peripheral blood of IBD patients $(18,19)$. The main cytokine appears to be IL-6, which displayed a serum concentration negatively correlated with BMD (19). The RANKL also is stimulated in these patients. Plasma levels of OPG were found to be elevated 2.4-fold in Crohn's disease and 1.9-fold in ulcerative colitis, probably representing an attempted response to reverse RANKL increased (18).

Both the American Gastroenterological Association (AGA) and British Society of Gastroenterology recommend the early use of immunomodulators in order to keep minimum dose of GC $(23,24)$. Patients in remission had higher BMD Z-scores than individuals with active disease, and this difference correlated with length of remission, approached normal after 3 years of remission (25). Moreover, patients treated with azathioprine had significantly increased BMD Z-scores which correlated to the drug's ability to induce disease remission rather than a direct effect of the drug.

\section{CELIAC DISEASE}

Celiac disease is a chronic inflammatory condition of the small intestine induced by dietary gluten in subjects who are genetically-susceptible. Over $75 \%$ of untreated patients with this disease present with low BMD. Therefore, it is considered one of the most frequent predisposing conditions to osteoporosis (26). Bone turnover markers are altered in patients with untreated celiac disease, reflecting reduction of bone formation and increase of resorption (10). Risk of fracture in patients with celiac disease may be only modestly elevated compared to the general population, probably due to rapid gains in bone mass with gluten-free diet (10).

The two main mechanisms by which celiac disease causes osteoporosis are systemic inflammation and intestinal malabsorption. Malabsorption leads to general malnutrition, decreased bioavailability of calcium and vitamin D, lower body mass index (BMI) and reduced muscle mass $(10,26)$. Vitamin D deficiency and low calcium concentrations are presented in 30\%-60\% of these patients (26). Secondary hyperparathyroidism is often present due to hypovitaminosis $\mathrm{D}$, and lead to increased bone turnover (10).

\section{CYSTIC FIBROSIS}

Cystic fibrosis (CF) occurs due to a defect in the cystic fibrosis transmembrane conductance regulator, a chloride channel, resulting in viscous secretion in lungs, pancreas, intestine, biliary tract and male genital tract, which leads to inflammation, infection, tissue destruction and ultimately organ failure (10). The inflammation occurs in response to the chronic infection in the airways (27).

There is a particular concern of osteoporosis in this disease since lung transplantation is the main treatment but poor bone health is a contraindication for this therapy (10). Individuals with $\mathrm{CF}$, when compared to healthy subjects present with 100 -fold increased risk of vertebral fractures and a ten-fold increased risk of rib fractures, the two most common sites for fractures (27).

Low BMD is prevalent in $30 \%$ of these patients, especially in older, malnourished and not healthier individuals, although young age by itself is not protective. The most important factor related to osteoporosis is disease duration defined by lung function and nutritional parameters. Others factors include malabsorption (especially due to hypovitaminosis D and poor nutrition, deficits in lean body mass, physical inactivity, delayed puberty, hypogonadism, and chronic pulmonary inflammation, besides GC therapy $(10,28)$. Although calcium absorption can be normal if hypovitaminosis D is not presented, endogenous fecal losses are significantly increased, thereby affecting net calcium retention in bone.

Serum IL-6, IL-1, TNF- $\alpha$, and M-CSF are increased in those with exacerbated lung infections (10). NTX, IL-6, TNF- $\alpha$, and C-reactive protein concentrations decrease after successful antibiotic therapy, and osteocalcin increases. Another evidence of the role of inflammation is osteoporosis related to $\mathrm{CF}$ is the link between C-reactive protein and severity of bone disease (28). Serum IGF-1, a bone anabolic factor, is frequently decreased in patients with CF probably due to both malnutrition and inflammatory cytokines which causes growth hormone insensitivity (10). 


\section{CHRONIC OBSTRUCTIVE PULMONARY DISEASE (COPD)}

Although primarily a respiratory disease, COPD may present with systemic complications including osteoporosis, even in the absence of GC use $(29,30)$. Low bone mass can appear even in mild severity diseases. McEvoy and cols. (31) observed that vertebral fractures were present in up to $50 \%$ steroid naive males with COPD. A Osteoporotic Fractures in Men study also demonstrated a 2 -fold increased risk of vertebral osteoporosis compared to men with no history of COPD or asthma independent of age, BMI, and smoking (32).

Increased circulating concentrations of IL-6, TNF- $\alpha$ and CRP are found in COPD patients, even if the disease is stable, and they are associated with presence of low bone mass $(29,30)$.

\section{DIAGNOSIS}

In evaluation of osteoporotic patients, medical history and physical examination must be performed to look for secondary causes of osteoporosis. History of arthralgia should always be asked about and osteoarticular examination always performed to exclude rheumatologic disease. Erythrocyte sedimentation rate has to be verified to exclude inflammatory diseases (33). If erythrocyte sedimentation rate is increased, other rheumatologic serum markers such as rheumatoid factor and anti-nuclear antibody can be requested. History of diarrhea should be evaluated in order to exclude celiac disease and IBD, but sometimes celiac disease can be present even without diarrhea and loss of weight should be considered.

The indication of dual energy X-ray absorptiometry (DXA) is controversial. Although guidelines of the International Society for Clinical Densitometry (ISCD) indicate DXA whenever patients have a disease related to high fracture risk (34), the societies of Rheumatology, Gastroenterology and Pneumology do not recommend DXA for all these patients, once many of them have normal exams.

For SLE patients, DXA is recommended for postmenopausal women with previous fragility fracture, or those aged 60 years or older with a risk factor for osteoporosis, or older than 65 years without a risk factor. No guidelines exist for premenopausal women or postmenopausal women younger than 65 years old, but some authors agree that if these patients have a high level of systemic inflammatory disease, they are prob- ably at an increased risk for fracture and a BMD measurement might be appropriate (35).

For IBD patients, the American Gastroenterological Association published guidelines that recommend DXA if they have another risk factor for osteoporosis such as history of low trauma fracture, postmenopause or hypogonadism, male older than 50 years of age or chronic GC therapy. The American Gastroenterological Association recommends that if DXA is normal it should be repeated in 2-3 years (22). According to some authors there is no justification for DXA screening in patients with celiac disease due to its reversibility with gluten-free diet (14).

The Cystic Fibrosis Foundation Bone Health Consensus Committee recommends determining a baseline BMD in children older than 8 years old with other risk factors for poor bone health such as low BMI (ideal BMI $<90 \%$ ), active lung disease (FEVI $<50 \%$ predicted), GC $\geq 5 \mathrm{mg}$ /day for more than 3 months/year, delayed puberty, or history of fractures. DXA should be obtained in all patients at the age of 18 years due to the high risk of fracture (36).

The $\mathrm{Z}$ score, which reflects the comparison with $\mathrm{BMD}$ of healthy individuals of the same age, is preferred in premenopausal women as it reflects better the effect of a secondary cause of osteoporosis than T-scores do (34). If the patients present with a Z-score lower than -2 SD a complete evaluation should be performed: inflammatory disease activity status should be assessed (inflammatory markers such erythrocyte sedimentation rate and C-reactive protein which is related to the activity of the disease), nutritional evaluation, complete blood count and albumin; bone metabolism exams, including serum concentrations of alkaline phosphatase and/or CTX (c-telopeptides of type 1 collagen), serum and urinary calcium, creatinine, 25-hydroxyvitamin D (25OHD) and PTH. If there are symptoms of gonadal failure, total and free testosterone should be tested in males. As auto-immune diseases are associated thyroidfunction tests should be performed to exclude subclinical hyperthyroidism (14).

\section{TREATMENT}

\section{General recommendations}

Firstly, the baseline inflammatory status should be corrected as effectively as possible, in order to lower disease activity and therefore the production of pro-inflammatory cytokines. Sometimes this is only obtained 
with high doses of GC, and prevention of GC-induced osteoporosis should be implemented as a guideline recommendation including the lowest dose possible (9).

Other factors, risk of osteoporosis, and fracture should be addressed. Therefore, the nutritional state should be improved and physical activity optimized to tolerance, which presuppose the evaluation and treatment of muscle weakness (10).

\section{Corrections of complementary disturbances}

Serum and urinary calcium, serum 25OHD, and PTH concentrations should be used to evaluate hypovitaminosis $\mathrm{D}$, secondary hyperparathyroidism and low net calcium balance. In randomized trials, the use of calcium and vitamin $\mathrm{D}$ alone had no significant benefit in bone density (10). However, these disturbances usually found in inflammatory diseases should be corrected to avoid the interference with anti-osteoporotic treatment efficacy. Calcium intake (from diet, added to supplementation) must be at least $1,200 \mathrm{mg} / \mathrm{d}$, and vitamin D supplementation should be at least $800 \mathrm{UI} / \mathrm{d}$ if any of those disturbances are found (10).

In CF patients it is recommended that serum vitamin D concentration should be monitored at least yearly in order to maintain a target of serum $25 \mathrm{OHD}$ higher than $25-30 \mathrm{ng} / \mathrm{mL}$ (35). In these patients, supplemental vitamin K may help the anti-osteoporotic treatment efficacy probably by promoting post-translational $\gamma$-carboxylation of osteocalcin which favors calcium binding in bone. In 20 children with $\mathrm{CF}$, vitamin $\mathrm{K}$ in a dose of $10 \mathrm{mg}$ /week for 1 year increased serum markers of bone formation, and decreased markers of bone resorption and serum PTH (37).

Growth hormone $(\mathrm{GH})$ can be used in prepubertal children and adolescents with significant growth failure and significant bone deficits (38-40).

\section{Bisphosphonates}

All medications involved in the inhibition of osteoclast activity may be helpful in inflammatory diseases, including bisphosphonates. This class of medication has already demonstrated its efficacy in GC-induced osteoporosis both as prevention and as treatment (9).

As with osteoclasts, macrophages are also sensitive to the action of bisphosphonates probably due to their common origin. These medications reduce the macrophage production of TNF- $\alpha$, IL- 1 and nitric oxide (NO) and induce apoptosis of monocyte-macrophage-derived cell lines. These effects are in part dependent on RANKL inhi- bition and in part dependent on cytoplasmic events which involve protein-kinase $\mathrm{C}$ and iron ions. Moreover, they reduce the production of oxygen free radicals by polymorphonuclear cells which are involved in the induction and enhancing of inflammatory response in RA, and reduce the production of different pro-inflammatory mediators and the expression of adhesion molecules in synovial lining cells. Bisphosphonates also have anti-neoangiogenic activity and therefore reduce lymphocyte and pro-inflammatory cell arrival at the articular region (4l). All these effects explain why, although bisphosphonates enhance proliferation of T-lymphocytes, they have been successfully used to treat bone loss secondary to RA (5). Many studies have shown that Alendronate and Zoledronate are able to reduce joint swelling and blood inflammatory tests (IL-1, IL-6, TNF- $\alpha$, b2-microglobulin and erythrocyte sedimentation rate and C-reactive protein values) in various experimental arthritis animal models and in human studies. Otherwise, these effects were only seen in high dosage of alendronate $(40 \mathrm{mg}$ /day intravenously and over 90 consecutive days) (41). In IBD, pamidronate, alendronate, and risedronate were effective in increasing BMD compared to placebo (42-45). The Cystic Fibrosis Foundation Bone Health Consensus Committee recommends the use of bisphosphonates in adults if BMD Z-score is lower than -2.0 , or if it is lower than -1.0 with history of fragility fractures, if they are awaiting lung transplant or experience BMD loss greater than 3\%-5\%/year (36).

\section{New medications}

Other new medications like recombinant Fc-osteoprotegerin (Fc-OPG), anti-RankL antibody, c-Src-inhibitors, cathepsin K, and the inhibitor of chloride channel CLC7 will probably prove very useful to treat osteoporosis in inflammatory disease, due to their mechanism of action.

Physiologically, the RANK/RANKL/OPG system is an obvious therapeutic target for the treatment of osteoporosis secondary to inflammatory disease. Denosumab is a fully human monoclonal anti-RANKL antibody. This medication blocks the binding of RANKL to RANK (46). Clinical trials with AR patients demonstrated that compared with placebo markers of bone resorption (serum CTX-I and procollagen type 1 intact N-terminal propeptide [PINP]) and cartilage loss (urine CTX-II) were significantly decreased (47), and BMDs in the lumbar spine, total hip, and trochanter were significantly increased (48) on denosumab therapy. One remarkable fact revealed is that denosumab group exhibited significantly less progression of bone erosions 
compared with placebo $(\mathrm{P}<0.019)(49)$. Certainly, this is an advantage over bisphosphonates, for which the clinical trials over the past two decades have repeatedly failed to show such effect in usual doses (50-52).

IL- 1 receptor antagonist and TNF- $\alpha$ antibody demonstrated their effectiveness for treating RA disease activity and also reduced secondary osteoporosis as well as joint destruction. Infliximab is an anti-TNF $\alpha$ chimeric antibody (anti-TNF- $\alpha \mathrm{mAb}$ ). It has been reported to have a significant beneficial effect on bone metabolism in adults with Crohn's disease $(53,54)$. Infliximab increases markers of bone formation and decreases markers of bone resorption, and it improves BMD both in lumbar spine and hip $(18,20)$. OPG also decreases, probably due to less RANKL production (17). Besides a direct effect on the inflammatory process, infliximab can also improve bone metabolism by indirect effects, since it improves general well-being, nutritional status and physical activity, and diminishes the need for GC therapy (20). However, it is important to balance between the risk-benefit of using anti-TNF $\alpha \mathrm{mAb}$ if the objective is focus on osteoporosis since more studies need to be performed in order to assess the effects on fracture and not solely on BMD or bone markers; besides, a significant minority of patients with RA does not respond to these anti-TNF agents. Furthermore, they are associated with substantial risk of toxicity, require parenteral administration, and are extremely expensive (55). Interestingly, the combinatorial use of methotrexate (MTX) with infliximab (anti-TNF- $\alpha$ $\mathrm{mAb}$ ) or with etarnercept (TNF receptor immunoglobulin protein) significantly repairs bone erosion seen in RA patients within 2 years (7). Furthermore, although the combination of MTX and etanercept was effective for about $70 \%$ of RA patients the improvement of joint erosion was observed in $95 \%$ of patients, indicating that blocking TNF- $\alpha$ inhibits bone loss more than synovial inflammation through different pathways (7).

Other medications such as Anakinra, arecombinant human IL-1 receptor antagonist; Tocilizumab, a human anti-IL-6 receptor antibody; Vitaxin, an anti-integrin $\alpha v \beta 3$ $\mathrm{mAb}$; and Lect2, a novel cytokine need more studies to establish their effect in bone mass and fracture risk $(7,55,56)$.

\section{CONCLUSION}

Inflammation unquestionably has a major influence on bone metabolism leading to increase of resorption and fracture risk.
More studies addressing routine evaluation and treatment options are needed in order to define better guidelines for clinicians that treat these patients.

Bisphosphonates are nowadays the best choice of therapy for osteoporosis secondary to inflammation. Otherwise, the understanding of the action of leukocytes and cytokines in bone mass is extremely important in the development of new drugs that positively affect bone metabolism and may prove more powerful to treat bone loss associated to inflammatory diseases.

Disclosure: Dr. Paulo Lacativa has participated in Clinical Trials sponsored by AMGEN which involved one of the drugs cited in the text. Dr. Maria Lúcia reports no conflict of interest.

\section{REFERENCES}

1. Mundy GR. Osteoporosis and inflammation. Nutr Rev. 2007;65(12 Pt 2):S147-51.

2. Romas E, Gillespie MT, MartinT. Involvement of receptor activator of NFkappaB ligand and tumor necrosis factor-alpha in bone destruction in rheumatoid arthritis. Bone. 2002;30:340-6.

3. Teitelbaum SL. Osteoclasts: what do they do and how do they do it? Am J Pathol. 2007;170(2):427-35.

4. Udagawa N,Takahashi N, AkatsuT, et al. Origin of osteoclasts: mature monocytes and macrophages are capable of differentiating into osteoclasts under suitable microenvironment prepared by bone marrowderived stromal cells. Proc Natl Acad Sci U S A. 1990;87:7260-4.

5. Gillespie MT. Impact of cytokines and Tymphocytes upon osteoclast differentiation and function. Arthritis Res Ther. 2007;9:103.

6. Weitzmann MN, Pacifici R. The role of Iymphocytes in bone metabolism. Immunol Rev. 2005;208:154-68.

7. TanakaY, Nakayamada S, Okada Y. Osteoblasts and osteoclasts in bone remodeling and inflammation. Curr Drug Targets Inflamm Allergy. 2005;4(3):325-8.

8. Papanicolaou DA, Wilder RL, Manolagas SC, Chrousos GP. The pathophysiologic roles of interleukin-6 in human disease. Ann Intern Med. 1998;128(2):127-37.

9. Gregório LH, Lacativa PG, Melazzi AC, Russo LA. Glucocorticoid-induced osteoporosis. Arq Bras Endocrinol Metab. 2006;50(4):793-801.

10. Viswanathan A, Sylvester FA. Chronic pediatric inflammatory diseases: effects on bone. Rev Endocr Metab Disord. 2008;9(2):107-22.

11. Rodríguez-Bores L, Barahona-Garrido J, Yamamoto-Furusho JK. Basic and clinical aspects of osteoporosis in inflammatory bowel disease. World J Gastroenterol. 2007;13(46):6156-65.

12. Roldán JF, Del Rincón I, Escalante A. Loss of cortical bone from the metacarpal diaphysis in patients with rheumatoid arthritis: independent effects of systemic inflammation and glucocorticoids. J Rheumatol. 2006;33(3):508-16.

13. Seriolo B, Ferretti V, Sulli A, Caratto E, Fasciolo D, Cutolo M. Serum osteocalcin levels in premenopausal rheumatoid arthritis patients. Ann NY Acad Sci. 2002;966:502-7.

14. Lane NE. Therapy Insight: osteoporosis and osteonecrosis in systemic lupus erythematosus. Nat Clin Pract Rheumatol. 2006;2(10):562-9.

15. Borba VZ, Matos PG, Silva Viana PR, et al. High prevalence of vertebral deformity in premenopausal systemic lupus erythematosus patients. Lupus. 2005;14(7):529-33. 
16. Pineau CA, Urowitz MB, Fortin PJ, Ibanez D, Gladman DD. Osteoporosis in systemic lupus erythematosus: factors associated with referral for bone mineral density studies, prevalence of osteoporosis and factors associated with reduced bone density. Lupus. 2004;13(6):436-41.

17. Borba VZC, Vieira JGH, Kasamatsu T, Radominski SC, Sato El, Lazaretti-Castro M. Vitamin D deficiency in patients with active systemic lupus erythematosus. Osteoporos Int. 2009;20(3):427-33.

18. Ali T, Lam D, Bronze MS, Humphrey MB. Osteoporosis in inflammatory bowel disease. Am J Med. 2009;122(7):599-604.

19. Paganelli $\mathrm{M}$, Albanese $\mathrm{C}$, Borrelli $\mathrm{O}$, et al. Inflammation is the main determinant of low bone mineral density in pediatric inflammatory bowel disease. Inflamm Bowel Dis. 2007;13(4):416-23.

20. Mauro M, Radovic V, Armstrong D. Improvement of lumbar bone mass after infliximab therapy in Crohn's disease patients. Can J Gastroenterol. 2007;21(10):637-42.

21. Souza HN, Lora FL, Kulak CAM, Mañas NCP, Amarante HMB, Borba VZC. Low levels of 25-hydroxyvitamin D (25OHD) in patients with inflammatory bowel disease and its correlation with bone mineral density. Arq Bras Endocrinol Metab. 2008;52(4):684-91.

22. Ashcroft AJ, Cruickshank SM, Croucher PI, et al. Colonic dendritic cells, intestinal inflammation, and T cell-mediated bone destruction are modulated by recombinant osteoprotegerin. Immunity. 2003;19:849-61.

23. Lichtenstein GR, Abreu MT, Cohen R, Tremaine W; American Gastroenterological Association Institute medical position statement on corticosteroids, immunomodulators, and infliximab in inflammatory bowel disease. Gastroenterology. 2006;130:935-9.

24. Carter MJ, Lobo AJ, Travis SPL. Guidelines for the management of inflammatory bowel disease in adults. Gut. 2004;53(Suppl V):v1-16.

25. Reffitt DM, Meenan J, Sanderson JD, et al. Bone density improves with disease remission in patients with inflammatory bowel disease. Eur J Gastroenterol Hepatol. 2003;15(12):1267-73.

26. Cashman KD. Altered bone metabolism in inflammatory disease: role for nutrition. Proc Nutr Soc. 2008;67(2):196-205.

27. Elborn JS. How can we prevent multisystem complications of cystic fibrosis? Semin Respir Crit Care Med. 2007;28(3):303-11.

28. Shead EF, Haworth CS, Gunn E, Bilton D, Scott MA, Compston JE. Osteoclastogenesis during infective exacerbations in patients with cystic fibrosis. Am J Respir Crit Care Med. 2006;174(3):306-11.

29. Sabit R, Bolton CE, Edwards PH, et al. Arterial stiffness and osteoporosis in chronic obstructive pulmonary disease. Am J Respir Crit Care Med. 2007;175(12):1259-65.

30. Sevenoaks MJ, Stockley RA. Chronic obstructive pulmonary disease, inflammation and co-morbidity - a common inflammatory phenotype? Respir Res. 2006;7:70.

31. McEvoy CE, Ensrud KE, Bender E, et al. Association between corticosteroid use and vertebral fractures in older men with chronic obstructive pulmonary disease. Am J Respir Crit Care Med. 1998;157:704-9.

32. Dam TT, Harrison S, Fink HA, Ramsdell J, Barrett-Connor E; for the Osteoporotic Fractures in Men (MrOS) Research Group. Bone mineral density and fractures in older men with chronic obstructive pulmonary disease or asthma. Osteoporos Int. Epub 2009 Oct 9.

33. Lacativa PG, de Farias ML. Office practice of osteoporosis evaluation. Arq Bras Endocrinol Metab. 2006;50(4):674-84.

34. 2007 ISCD Official Positions. Available from: http://www.iscd.org/Visitors/pdfs/ISCD2007OfficialPositions-Combined-AdultandPediatric.pdf

35. Recommendations for the prevention and treatment of glucocorticoid-induced osteoporosis: 2001 update. American College of Rheumatology Ad Hoc Committee on Glucocorticoid-Induced Osteoporosis. Arthritis Rheum. 2001;44(7):1496-503.

36. Aris RM, Merkel PA, Bachrach LK, et al. Guide to bone health and disease in cystic fibrosis. J Clin Endocrinol Metab. 2005;90(3):1888-96.
37. van Hoorn JH, Hendriks JJ, Vermeer C, Forget PP. Vitamin K supplementation in cystic fibrosis. Arch Dis Child. 2003;88 (11):974-5.

38. Hardin DS, Sy JP. Effects of growth hormone treatment in children with cystic fibrosis: the National Cooperative Growth Study experience. J Pediatr. 1997;131(1 Pt 2):S65-9.

39. Hardin DS, Ahn C, Prestidge C, Seilheimer DK, Ellis KJ. Growth hormone improves bone mineral content in children with cystic fibrosis. J Pediatr Endocrinol Metab. 2005;18(6):589-95.

40. Hardin DS, Adams-Huet B, Brown D, et al. Growth hormone treatment improves growth and clinical status in prepubertal children with cystic fibrosis: results of a multicenter randomized controlled trial. J Clin Endocrinol Metab. 2006;91(12):4925-9.

41. Corrado A, Santoro N, Cantatore FP. Extra-skeletal effects of bisphosphonates. Joint Bone Spine. 2007;74(1):32-8.

42. Haderslev KV, Tjellesen L, Sorensen HA, Staun M. Alendronate increases lumbar spine bone mineral density in patients with Crohn's disease. Gastroenterology. 2000;119:639-46.

43. Henderson S, Hoffman N, Prince R. A double-blind placebo controlled study of the effects of the bisphosphonate risedronate on bone mass in patients with inflammatory bowel disease. Am J Gastroenterol. 2006;101:119-23.

44. Bartram SA, Peaston RT, Rawlings DJ, Francis RM, Thompson NP. A randomized controlled trial of calcium with vitamin $\mathrm{D}$, alone or in combination with intravenous pamidronate, for the treatment of low bone mineral density associated with Crohn's disease. Aliment PharmacolTher. 2003;18:1121-7.

45. Stokkers PC, Deley M, Van Der Spek M, Verberne HJ, Van Deventer SJ, Hommes DW. Intravenous pamidronate in combination with calcium and vitamin D: highly effective in the treatment of low bone mineral density in inflammatory bowel disease. Scand J Gastroenterol. 2006;41:200-4.

46. McClung M. Role of RANKL inhibition in osteoporosis. Arthritis ResTher. 2007;9(Suppl 1):S3.

47. Lane NE, lannini $M$, Atkins $C$, et al. RANKL inhibition with denosumab decreases markers of bone and cartilage turnover in patients with rheumatoid arthritis. Arthritis Rheum. 2006;54:S225.

48. Dore R, Hurd E, Palmer W, et al. Denosumab increases bone mineral density in patients with rheumatoid arthritis. Arthritis Rheum. 2006;54:S240.

49. Cohen SB, Valen P, Ritchlin CT, et al. RANKL inhibition with denosumab reduces progression of bone erosions in patients with rheumatoid arthritis: month $6 \mathrm{MRI}$ results. Arthritis Rheum. 2006;54:S831.

50. Ralston SH, Hacking L, Willocks L, Bruce F, Pitkeathly DA. Clinical, biochemical, and radiographic effects of aminohydroxypropylidene bisphosphonate treatment in rheumatoid arthritis. Ann Rheum Dis. 1989;48:396-9.

51. Maccagno A, Di Giorgio E, Roldan EJ, Caballero LE, Perez Lloret $A$. Double blind radiological assessment of continuous oral pamidronic acid in patients with rheumatoid arthritis. Scand J Rheumatol. 1994;23:211-4.

52. Eggelmeijer F, Papapoulos SE, van Paassen HC, et al. Increased bone mass with pamidronate treatment in rheumatoid arthritis. Results of a three-year randomized, double-blind trial. Arthritis Rheum. 1996;39:396-402.

53. Ryan BM, Russel MGV, Schurgers L, et al. Effect of antitumour necrosis factor - a therapy on bone turnover in patients with active Crohn's disease: a prospective study. Aliment PharmacolTher. 2004;20:851-7.

54. Bernstein M, Irwin S, Greenberg GR. Maintenance infliximab treatment is associated with improved bone mineral density in Crohn's disease. Am J Gastroenterol. 2005;100:2031-5.

55. Brown MA. Antibody treatments of inflammatory arthritis. Curr Med Chem. 2005;12(25):2943-6.

56. Krause $\mathrm{KH}$, Clark RA. Geneva Biology of Ageing Workshop 2000: phagocytes, inflammation, and ageing. Exp Gerontol. 2001;36(2):373-81. 